\title{
A simple faulted phase-based fault distance estimation algorithm for a loop distribution system
}

\author{
Shwe Myint, Warit Wichakool \\ Department of Electrical Engineering, Faculty of Engineering, Prince of Songkla University, Songkhla, Thailand
}

\begin{tabular}{|c|c|}
\hline Article Info & ABSTRACT \\
\hline Article history: & This paper presents a single ended faulted phase-based traveling wave fault \\
\hline Received Jan 25, 2021 & localization algorithm for loop distribution grids which is that the sensor can \\
\hline Revised Nov 19, 2021 & localization. This localization algorithm uses a band pass filter to remove \\
\hline Accepted Nov 23, 2021 & $\begin{array}{l}\text { noise from the corrupted signal. The arriving times of the faulted phase- } \\
\text { based filtered signals can be obtained by using phase-modal and discrete }\end{array}$ \\
\hline Keywords: & $\begin{array}{l}\text { wavelet transformations. The estimated fault distance can be calculated } \\
\text { using the traveling wave method. The proposed algorithm presents detail }\end{array}$ \\
\hline Band pass filter & level analysis using three detail levels coefficients. The proposed algorithm \\
\hline Discrete wavelets transform & $\begin{array}{l}\text { is tested with MATLAB simulation single line to ground fault in a } 10 \mathrm{kV} \\
\text { grounded loop distribution system. The simulation result shows that the }\end{array}$ \\
\hline Fault location & faulted phase time delay can give better accuracy than using conventional \\
\hline Loop distribution system & time delays. The proposed algorithm can give fault distance estimation \\
\hline Noise & accuracy up to $99.7 \%$ with $30 \mathrm{~dB}$ contaminated signal-to-noise ratio (SNR) \\
\hline Traveling wave & for the nearest lines from the measured terminal. \\
\hline
\end{tabular}

This is an open access article under the CC BY-SA license.

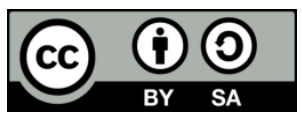

\section{Corresponding Author:}

Warit Wichakool

Department of Electrical Engineering, Faculty of Engineering, Prince of Songkla University

Hat Yai, Songkhla 90112, Thailand

Email: warit.wi@psu.ac.th

\section{INTRODUCTION}

A fault in a power system interrupts a modern way of living. An ability to locate a fault in a power system loop distribution network is very important to improve reliability for customer satisfaction and utilities. The outage location is needed to know accurately to remove the outage line quickly from the remaining parts of the system. Therefore, different conventional fault distance estimation methods such as impedance-based method, frequency domain method, and traveling wave (TW) method are used to solve this problem.

Some researchers utilized impedance-based algorithms with the help of system fault signals [1], [2]. Next, the knowledge-based method has been used to detect the fault location using neural networks, decision trees and support vector machine techniques [3]-[7]. Some previous researcher used voltage sag matching algorithm in [8], comparing faulty phase voltage with measure value approach was used in [9] and weighted least square approach in [10] respectively. All the above algorithms require training data sets with predefined different fault situations.

Traveling wave-based algorithms can give the best accuracy with the help of discrete wavelet transforms (DWT) filters. Most of fault localization algorithms use the measured fault signals from single end of the system by identifying the first sudden changing point in the waveform [11]-[13]. This method was analyzing in time domain that cannot get accurate arriving time for high impedance faults. A simple fault localization algorithm based on characteristics of network topology and recloser-generating traveling wave 
signals was introduced in [14]. However, the feeders without recloser cannot be protected by this algorithm. The combination of impedance and traveling wave-based algorithms are proposed [15]-[17]. The arriving times of zero and aerial components are extracted by DWT filter (db4, level1) and utilized to estimate fault distance [15]. Dijkstra shortest path algorithm was proposed based on arrival time of initial voltage traveling wave fronts at the two terminals [16]. However, these algorithms did not consider about fault section to trip correctly the faulty part. Moreover, these methods utilized the general aerial mode arriving time of modal components which cannot give the accurate fault information for all fault types.

A decision tree aided traveling wave fault location algorithm was introduced in [18] and this algorithm used the two adjacent arrival times of the faulted line. This method could not get highest accuracy because it used the arriving time of general aerial mode components of Clark's transform which cannot give the accurate fault information for all fault types. In addition, some single line to ground TW based methods used DWT filter and support vector regression (SVR) model [19], polarities of initial voltage and current signals [20], the difference of velocities by separating the initial arriving times of zero and aerial mode components [21] and the last one based on zero-sequence components distribution characteristics [22], respectively. These methods also used arriving time of conventional aerial mode that cannot give correct fault information for all faulted phases and fault types. The next method is asynchronous voltage-based TW data fusion method [23]. This method requires the data training. Moreover, a single phase to ground fault localization method [24] used the fault characteristics include in zero sequence voltages and currents. However, the method is suitable for radial distribution systems.

According to literature reviews, the best accuracy can be achieved based on the arrival time difference between the modal components of the current waveform monitored at the substation bus [19]. All the traveling wave methods applied in the above literature used the arriving time of conventional aerial mode components and only for transmission line and radial network topologies. However, the arriving time of these components cannot give the accurate and correct fault information and significantly one of the difficulties in loop distribution network is that the sensor can get many reflected signals from the fault point to face the complexity of detection of actual faulted length. Therefore, the proposed algorithm can choose the correct faulted section in [25], the accurate faulted path and uses the arriving time of faulted phase-based modal component [26] that can give higher accuracy than that of conventional methods with the consideration of noise effects, such as white noise. The paper is organized as section: 2 presents traveling wave method and modal transformation, research methodology is explained in section 3. Section 4 focus on simulation results and discussions for loop distribution test systems. The last section is conclusion.

\section{THEORY BACKGROUDS}

In this paper, the traveling wave reflected signal is mainly used to detect the arriving time of fault signal. These reflected signals are necessary to be significant and accurate. Therefore, the reflected signals are pass through the discrete wavelet filter via the phase signals are needed to be transformed using phase to modal transformation matrix to avoid mutual effects. The following subsections are theories and detail information of the component's background.

\subsection{Traveling wave theory}

In the proposed algorithm, the recorded fault signals from the measure bus are transformed to modal components. Different modes have different wave velocity depending on the system characteristic impedance. Hence, the arrival time of difference mode will result in a time delay. The location of singleended faults is given as (1) [24].

$$
d=\frac{v_{0} v_{1}\left(t_{2}-t_{1}\right)}{\left(v_{1}-v_{0}\right)}
$$

The time delay between zero mode current and aerial mode currents of Park's transformation is shown in (2). The time delay between zero mode current and modal difference currents is shown in (3) and the time delay between the zero mode and aerial mode currents of the Karenbauer's transformation in (4).

$$
\begin{aligned}
& d=\frac{v_{0} v_{1}\left(t_{2}-t_{1}\right)}{\left(v_{1}-v_{0}\right)} \\
& d=\frac{v_{0} v_{1}\left(t_{2}-t_{1}\right)}{\left(v_{1}-v_{0}\right)} \\
& d=\frac{v_{0} v_{1}\left(t_{2}-t_{1}\right)}{\left(v_{1}-v_{0}\right)}
\end{aligned}
$$


The variable $t_{0}$ is the arriving time of the zero mode, which can be obtained using the residual current. The value can be calculated from (5).

$$
d=\frac{v_{0} v_{1}\left(t_{2}-t_{1}\right)}{\left(v_{1}-v_{0}\right)}
$$

The zero-mode wave velocity can be estimated based on the system characteristic impedance,

$$
d=\frac{v_{0} v_{1}\left(t_{2}-t_{1}\right)}{\left(v_{1}-v_{0}\right)}
$$

where $L_{0}$ is the zero-sequence inductance and $C_{0}$ is zero sequence capacitance of the power line. The arriving time of the aerial mode using different transformation can be calculated using the same in (7).

$$
d=\frac{v_{0} v_{1}\left(t_{2}-t_{1}\right)}{\left(v_{1}-v_{0}\right)}
$$

The wave velocity of the aerial mode is given by (8),

$$
d=\frac{v_{0} v_{1}\left(t_{2}-t_{1}\right)}{\left(v_{1}-v_{0}\right)}
$$

where $L_{1}$ and $C_{1}$ are positive sequence inductance and capacitance of the power line respectively. The arriving time of the aerial mode can be different depending on the transformation used to process the time domain signal. Using these different arriving times of aerial components can result different accuracies; therefore, the modal difference component and faulted phase-based arriving time are used and compared with that of the conventional aerial mode components.

\subsection{Phase to modal transform}

The phase components are transformed to modal components using Karenbauer's transform to avoid mutual effects. The transformation matrix is shown in (9). The $\alpha, \beta$ and $\gamma$ modal represent the difference between two current phases. On the other hand, the zero-modal (0) is similar to the zero component in the sequence network.

$$
d=\frac{v_{0} v_{1}\left(t_{2}-t_{1}\right)}{\left(v_{1}-v_{0}\right)}
$$

In this article, the most accurate arriving time of aerial mode is chosen from the aerial components related with Park's, Clark's and Karenbauer's transform, and aerial different component.

\subsection{Wavelet transforms}

The DWT splits into two components. They are approximation coefficients (cA) (low frequency) and detail coefficients $(\mathrm{cD})$ (high frequency). Given an input signal x(k), its DWT can be calculated as (10),

$$
d=\frac{v_{0} v_{1}\left(t_{2}-t_{1}\right)}{\left(v_{1}-v_{0}\right)}
$$

where $a_{0}$ is the scale factor, $b_{0}$ is the translation factor. The variable $k$ represents an input sample number. Variables $m$ and $n$ represent mother wavelet and decomposed level, respectively.

In this paper, detail coefficients of db6 mother wavelet for level1, 2 and 3 are extracted and utilized to reconstruct the signals. The arriving times are detected from the component that is combining the three reconstructed signals using combinations of three detail levels, D1, D2 and D3 to obtain accurate arriving time of the reflected fault signal. The accuracy comparison of db4 and db6 was described in [20]. To estimate fault distance using traveling wave method, in this paper, faulted phase-based time delay is utilized. It is necessary to get correct faulted phase from the fault information accuracy and faulted line from previous work [20]. Figure 1 shows the three levels detail coefficients decomposition diagram. 


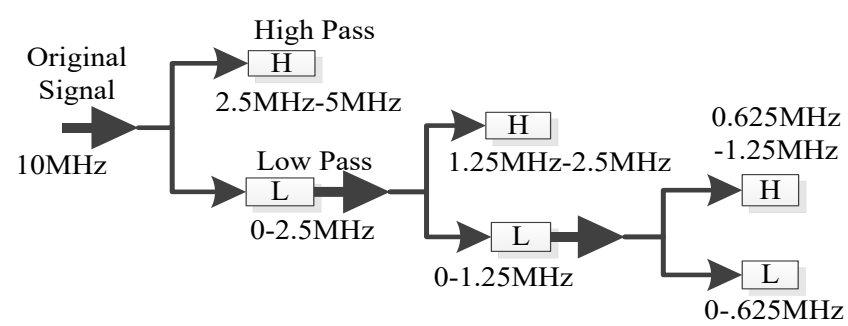

Figure 1. DWT decomposition

\section{PROPOSED ALGORITHM}

In this process, the recorded fault signals will firstly pass through the noise filter to remove noise from the input signal and then the filtered signals will be transformed to modal components to avoid mutual effects. In traveling wave fault location methods, the arriving times of fault signals are very important parameters to estimate fault distance, they can be extracted in time domain, sequence domain and wavelet domain as well. Therefore, the arriving times are extracted by using detail coefficients of DWT in this paper. After that, the correct faulted phase is detected by using fault information accuracy content in the three-phase current signals. Finally, the estimated fault distance can be estimated by using time delays of detail coefficients of modal current components. The process is generally illustrated in Figure 2 . The arriving times of absolute maxima of detail coefficient of zero and aerial mode current traveling wave signals can be estimated by using db6 mother wavelet because db6 mother wavelet can give more accurate arriving time than $\mathrm{db} 4$ mother wavelet that is used by most of previous fault location researchers for that purpose.

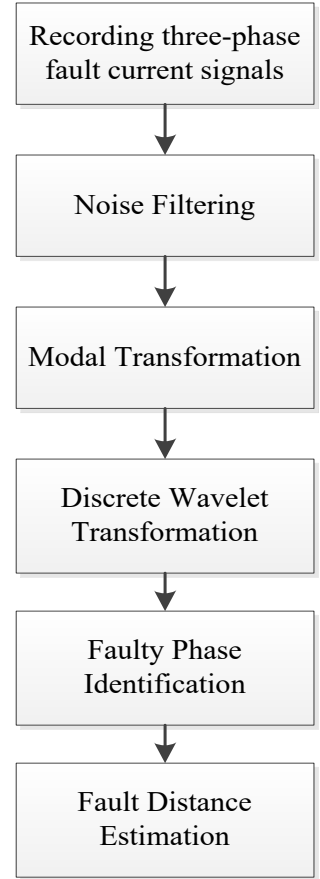

Figure 2. Fault estimation process

\section{SIMULATION RESULTS AND DISCUSSIONS}

This paper uses a loop overhead distribution (modified IEEE 14-bus) test system that consists of three power supplies of $33 \mathrm{kV} / 10 \mathrm{kV}, 50 \mathrm{~Hz}$ as shown in Figure 3. The faults are simulated in one kilometer step on all branches of the test system. This paper uses the distributed line parameter model in the simulation. Before locating fault, the system firstly identifies the faulted line with the help of the fault detectors at all buses. Therefore, the fault detectors are installed at all buses of the test system. In this paper, the faulted section identification algorithm [25] is applied to get accurate faulted feeder. After obtaining faulty feeder, the system continues to estimate fault distance using only the recording signals from bus 1 (B1) for all lines 
of the test system. Various fault distances with fault resistance $0.001 \Omega$ to $50 \Omega$ considering noise effects with 30 to 70 signal-to-noise ratio (SNR) dB are simulated and analyzed as well. Total 1650 cases for 55 points with 100 iterations for every case are studied. System parameters are generator source resistance $13.96 \Omega$ and source inductance of $0.35 \mathrm{H}$.

\subsection{Accuracy calculation}

In the loop distribution system, there are many possible predefined fault distance values because there are many paths from the fault location to the measure terminal. For example, when a fault occurs at the middle of line (L7) which is $4 \mathrm{~km}$ long, there are five possible ways to get different five arriving signals from the fault point $(F)$ to the measure terminal $\left(B_{1}\right)$. They are shown in the Figure 4.

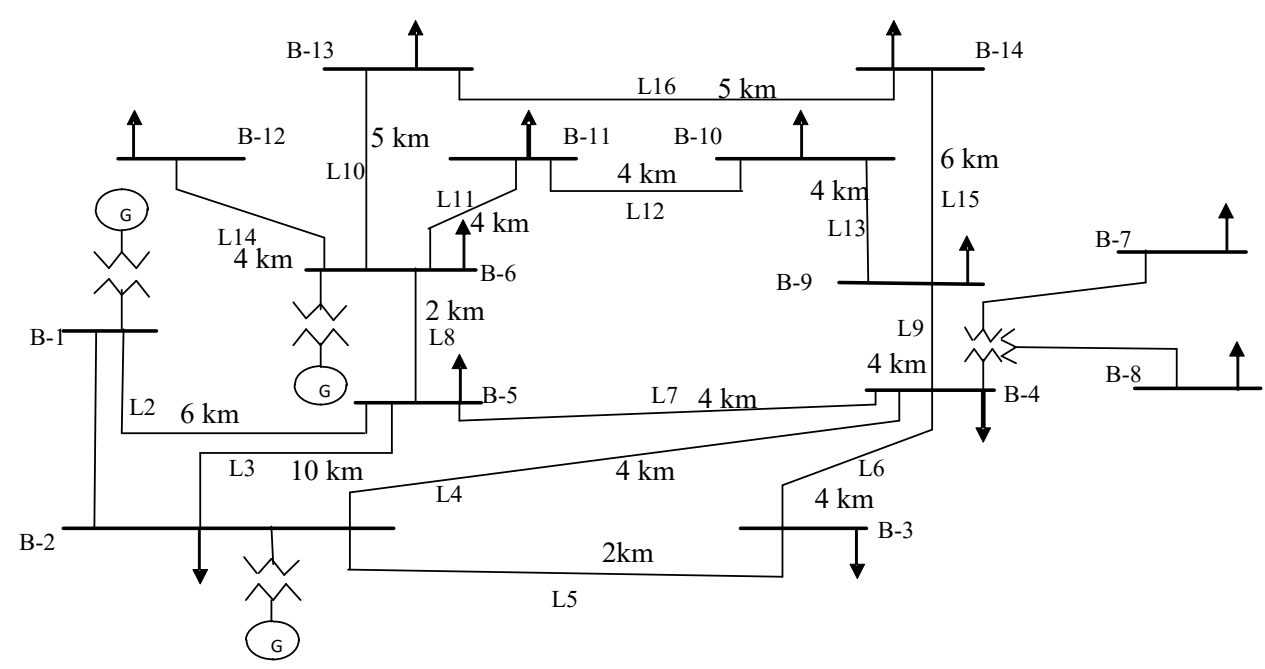

Figure 3. IEEE 14-Bus modified test system [25]

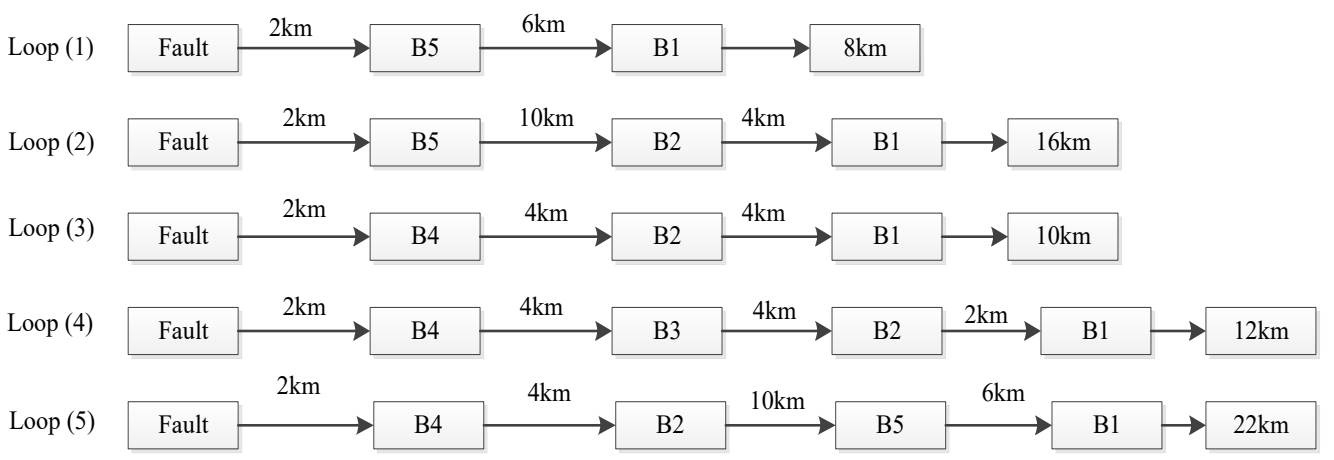

Figure 4. Possible actual fault distances when fault occurs at L7

According to these loops, possible fault distances from the measuring point are 8, 16, 10, 12 and $22 \mathrm{~km}$ that are possible actual fault distances for that case. Hence the range of possible fault distance (PFD) in this case is between 8 and $22 \mathrm{~km}$. If the estimated fault distance (EFD) resides within the possible fault distance, the estimated fault distance is feasible. Otherwise, the algorithm assumes that the estimated distance is erroneous and should be discarded. This scenario can occur under noisy environment. distance helps eliminate erroneous estimation. If the estimated fault distance is within the valid range, the algorithm can continue. Then we can access the performance of the algorithm by calculating the fault distance estimation accuracy (FDEAC) which can be defined by (11).

$$
d=\frac{v_{0} v_{1}\left(t_{2}-t_{1}\right)}{\left(v_{1}-v_{0}\right)}
$$


According to the example shown in Figure 4, the actual fault distance can have multiple values depending on the path the traveling wave take. Therefore, the algorithm must choose the possible fault distance from the list to calculate the accuracy value. In this paper, the value closest to the average estimated fault distance is chosen. If there are two possible AFD values, the smallest one is chosen.

For example, in the case of signals mixed with noise $60 \mathrm{SNR} \mathrm{dB}$, and the estimated fault distance is $7.9908 \mathrm{~km}$. Therefore, the actual fault distance would be $8 \mathrm{~km}$ because it is the closest one to the estimated value in this case. The FDEAC is $99.89 \%$ by (11).

\subsection{Arriving time based on faulted phase}

This paper uses the arrival time of the mode related to the faulted phase to achieve better accuracy. For example, the arrival times of the travelling wave when phase ' $c$ ' to ground (CG) fault occurs in the middle of L7. Four modes of travelling wave are extracted from the simulation and shown in Table 1.

From this example, the arrival time of $\beta$ and $\gamma$ modal components are the same because they are related to phase 'c', the faulted phase. If wrong information is used, the estimation could be erroneous. Therefore, the proposed algorithm used the arriving time of faulted phase related modal component to get higher accuracy.

Table 1. Arriving times of detail coefficients of zero and aerial mode components when a single line to

\begin{tabular}{cc}
\multicolumn{2}{c}{ ground (CG) fault occurs at L7 } \\
\hline Components & Arriving time (ms) \\
\hline$\alpha$ modal & 40.05 \\
$\beta$ modal & 40.03 \\
$\gamma$ modal & 40.03 \\
Zero modal & 40.15 \\
\hline
\end{tabular}

\subsection{Detail level analysis without noise}

In order to obtain the time delay, the wavelet transform is used to localize the arrival time among different modes. This paper uses the db6 mother wavelet and three level of decomposition to extract the arrival time information. To verify the best accuracy, different combinations of detail coefficients are used to compared the fault estimation error. The cases are the arriving times taken from: i) the combination of reconstructed signals by using detail coefficients level1 (D1), level2 (D2) and level3 (D3) shown here as $(\mathrm{D} 1+\mathrm{D} 2+\mathrm{D} 3)$; ii) the combination of reconstructed signals by using detail coefficients, D1 and D2, shown here as (D1+D2); iii) the reconstructed signals by using detail coefficients, D1; iv) the reconstructed signals by using detail coefficients, D2; and v) the reconstructed signals by using detail coefficients, D3, respectively. In this study, the proposed algorithm is tested with single line to ground (SLG) fault for all lines of test system. The result is shown in Table 2. Table 2 shows the performance of FDEAC for all lines of the test system without noise in the simulation. This accuracy using the time delay with combination of the fault information from the detail level 1,2 and $3\left(D_{1}+D_{2}+D_{3}\right)$ give the best accuracy in five comparative cases, which is expected because they cover widest frequency range of all combinations.

Table 2. Average \% of FDEAC for all lines with different detail levels without noise effects

\begin{tabular}{cccccc}
\hline \multirow{2}{*}{ Faulted Lines } & \multicolumn{5}{c}{ \% FDEAC by } \\
& $\mathrm{D}_{1}+\mathrm{D}_{2}+\mathrm{D}_{3}$ & $\mathrm{D}_{1}+\mathrm{D}_{2}$ & $\mathrm{D}_{1}$ & $\mathrm{D}_{2}$ & $\mathrm{D}_{3}$ \\
\hline L1 & 99.68 & 99.68 & 99.90 & 0.00 & 98.19 \\
L2 & 99.75 & 99.97 & 99.68 & 99.68 & 98.90 \\
L3 & 99.91 & 93.53 & 93.62 & 93.81 & 94.66 \\
L4 & 99.82 & 99.82 & 85.91 & 99.82 & 99.68 \\
L5 & 99.68 & 99.81 & 99.98 & 99.64 & 66.27 \\
L6 & 99.89 & 99.58 & 99.79 & 99.79 & 85.52 \\
L6 & 99.89 & 99.58 & 99.79 & 99.79 & 85.52 \\
L7 & 99.89 & 94.44 & 94.27 & 99.99 & 99.82 \\
L8 & 99.91 & 99.83 & 99.91 & 63.17 & 99.19 \\
L9 & 99.85 & 99.98 & 99.94 & 98.34 & 94.99 \\
L10 & 99.85 & 99.98 & 99.93 & 99.98 & 99.65 \\
L11 & 99.85 & 94.11 & 99.94 & 94.76 & 85.63 \\
L12 & 99.92 & 95.63 & 95.63 & 98.48 & 99.92 \\
L13 & 95.85 & 93.90 & 96.86 & 93.90 & 51.82 \\
L14 & 99.85 & 88.52 & 99.94 & 88.52 & 80.95 \\
L15 & 99.88 & 96.10 & 95.05 & 99.78 & 99.83 \\
L16 & 99.92 & 97.50 & 99.99 & 97.50 & 89.40 \\
Average & 99.59 & 97.02 & 97.52 & 95.14 & 97.05 \\
\hline
\end{tabular}




\subsection{Detail level analysis with noise}

In order to verify the accuracy of the set of detail coefficients used to extract the arrival time, the Gaussian white noise is added to simulate a practical situation where the input signal may be noisy. The simulation was done with SNR from 30 to $70 \mathrm{~dB}$. The result is shown in Figure 5 .

According to Figure 5, although the estimation accuracies by D1 are the best in overall, the values are not significant in single case for five parameters with different detail levels. However, the parameter with combination of detail level 1, level 2, and level 3 can give better accuracy in high noise levels, such as $40 \mathrm{~dB}$ and $50 \mathrm{~dB}$ and the fault distance estimation accuracy by using this parameter for most of faulted lines are above $99 \%$. Therefore, the arriving times for proposed fault distance estimation algorithm can be detected from the component that is a combination of reconstructed signals by using detail coefficients level 1 , level 2 and level $3(\mathrm{D} 1+\mathrm{D} 2+\mathrm{D} 3)$ of db6 mother wavelet.

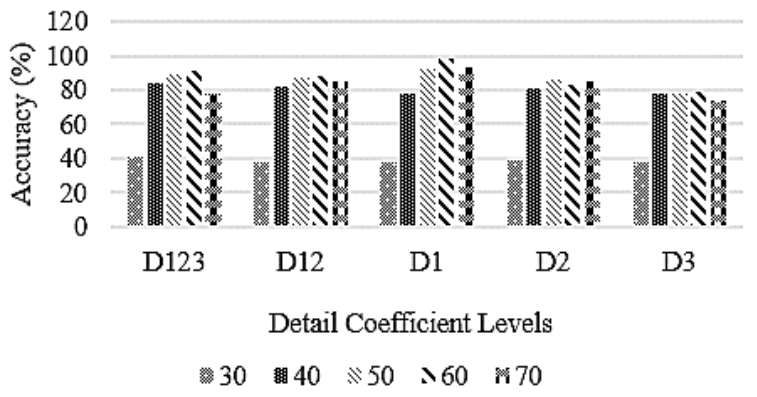

Figure 5. Performance of average FDEAC for all lines with different detail levels under different noise levels

\subsection{Accuracy performance of proposed algorithm}

Simulation results with additive noise of different signal-to-noise ratio are shown in Table 3 . The results compare the average accuracy of fault distance estimation for the travelling wave using different delay time, including dq, $\mathrm{d} 0$ and modal transformation. Examples include all single line to ground faults.

According to the result in Table 3 in the case of phase 'a' to ground (AG) fault, higher accuracy can be obtained from the time delay of the faulted phase, meaning that the algorithm will use the time delay from $\Delta t_{\alpha}$ or $\Delta t_{\beta}$. The estimation result using the non-faulted phase, specifically $\Delta t_{\gamma}$, g ive low accuracy percentage. In addition, the estimation accuracy using time delay from the faulted phase is comparable to the time delay from traditional $\mathrm{dq}$ and $\mathrm{d} 0$ transformation

In the case of phase ' $\mathrm{b}$ ' to ground (BG) fault, a very good average accuracy can be found using the faulted phase time delay $\left(\Delta t_{\alpha}\right.$ or $\Delta t_{\gamma}$ ) specifically above $99 \%$. There is exception at $30 \mathrm{~dB}$ SNR cases. The accuracy gets lower because of higher noise interference. Furthermore, if the conventional time delay, $\Delta t_{d}$, is used, the estimation error is less accurate for the BG fault.

Table 3. Average fault distance estimation accuracy percentage of proposed fault localization algorithm for fault occurred at L1

\begin{tabular}{ccccccc}
\hline Fault type & $\mathrm{SNR}(\mathrm{dB})$ & $\Delta t_{d q}$ & $\Delta t_{d}$ & $\Delta t_{\alpha}$ & $\Delta t_{\beta}$ & $\Delta t_{\gamma}$ \\
\hline AG & 30 & 99.88 & 99.96 & 99.49 & 99.98 & 72.63 \\
AG & 40 & 99.56 & 99.51 & 99.54 & 99.51 & 50.48 \\
AG & 50 & 99.28 & 99.28 & 99.28 & 99.28 & 32.31 \\
AG & 60 & 99.26 & 99.26 & 99.26 & 99.26 & 69.44 \\
AG & 70 & 99.26 & 99.26 & 99.26 & 99.26 & 55.33 \\
BG & 30 & 31.10 & 26.63 & 31.80 & 13.85 & 93.50 \\
BG & 40 & 98.05 & 47.93 & 94.05 & 13.85 & 99.15 \\
BG & 50 & 99.84 & 73.91 & 99.88 & 18.96 & 99.86 \\
BG & 60 & 99.63 & 91.27 & 99.66 & 89.67 & 99.66 \\
BG & 70 & 99.28 & 98.40 & 99.28 & 82.00 & 99.28 \\
CG & 30 & 97.87 & 99.56 & 40.04 & 99.64 & 98.48 \\
CG & 40 & 98.79 & 99.79 & 22.37 & 99.79 & 99.79 \\
CG & 50 & 99.10 & 99.26 & 61.25 & 99.26 & 99.26 \\
CG & 60 & 99.26 & 99.26 & 36.62 & 99.26 & 99.26 \\
CG & 70 & 99.26 & 99.26 & 49.10 & 99.26 & 99.26 \\
\hline
\end{tabular}


Finally, in the case of phase ' $c$ ' to ground (CG) fault, the fault distance estimation result using the information form the faulted phase $\left(\Delta t_{\beta}\right.$ or $\left.\Delta t_{\gamma}\right)$ gives above $99 \%$ accuracy. In addition, the proposed algorithm using the faulted phase time delay can get better accuracy than the conventional time delay under low signal-to-noise ratio ( $30 \mathrm{~dB}$ and $40 \mathrm{~dB})$. These results are similar to the (AG) fault cases.

According to the result shown in Table 3, the proposed algorithm has demonstrated that the time delay of that related to the faulted phase of the transform current waveform using Karenbauer's transformation give the best accuracy. Furthermore, in case of the single phase to ground fault, two timedelay parameters should be the very close, for example, $\Delta t_{\alpha}$ and $\Delta t_{\beta}$ for phase 'a' to ground fault. Therefore, the algorithm can use this information to verify the result as well.

To demonstrate an overall performance, the averaged fault distance accuracy percentage of all lines are shown in Figure 6. The results show that the performance of average fault distance estimation accuracy for all lines with additive noise at $70 \mathrm{~dB}$ SNR. The simulations were done for high fault current at $0.001 \mathrm{Ohm}$ fault resistance shown in Figure 6(a) and moderate fault resistance at 50 Ohms shown in Figure 6(b). Each subfigure shows average fault distance estimation accuracy using three-time delays: d0 time delay, dq time delay and faulted phase (FP) time delay to compare the overall accuracy.

According to the result in Figure 6, the proposed FP subscription time delay $\left(\Delta t_{\alpha}, \Delta t_{\beta}\right.$, and $\left.\Delta t_{\gamma}\right)$ gives better averaged accuracy than the conventional time delays $\left(\Delta t_{d}\right.$ and $\left.\Delta t_{d q}\right)$ for fault distance estimation in case of faults in most lines. The averaged estimation accuracy for fault on line 13 is worse than the algorithm that use the $\Delta t_{d q}$ time delay. However, this recording was done at Bus 1 , which is far away. Different point of observation can be done to double check or narrow down the fault location. The result on line 4 at $50 \mathrm{Ohms}$ fault resistance is also worse than the $0.001 \mathrm{Ohm}$ fault resistance case. This may be due to shorter distance and lower fault signal causing the wavelet to give the inaccurate time delay. However, overall performance is good for fault on all lines in this network topology. Hence, the time delay of the faulted phase should be used to calculate the fault distance.

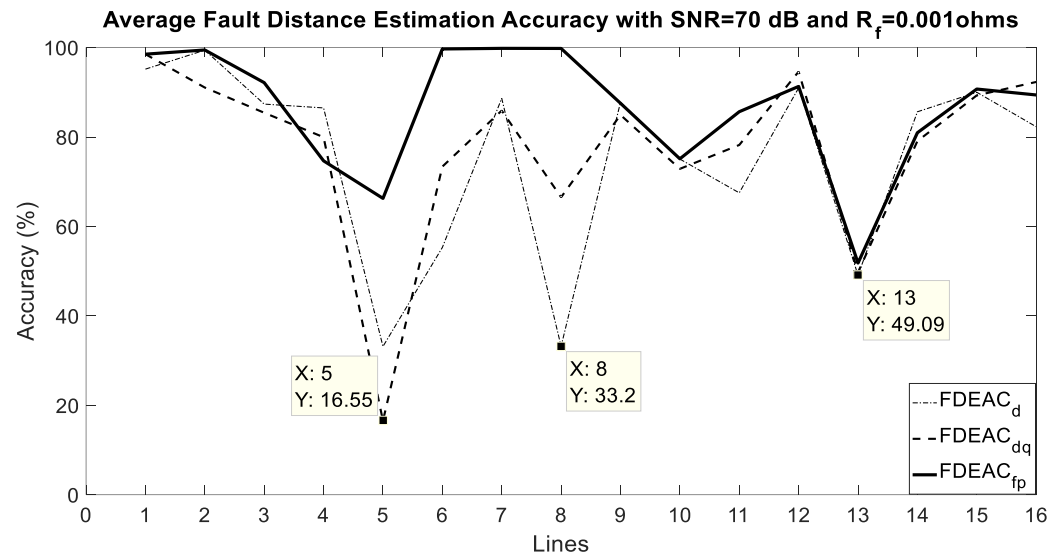

(a)

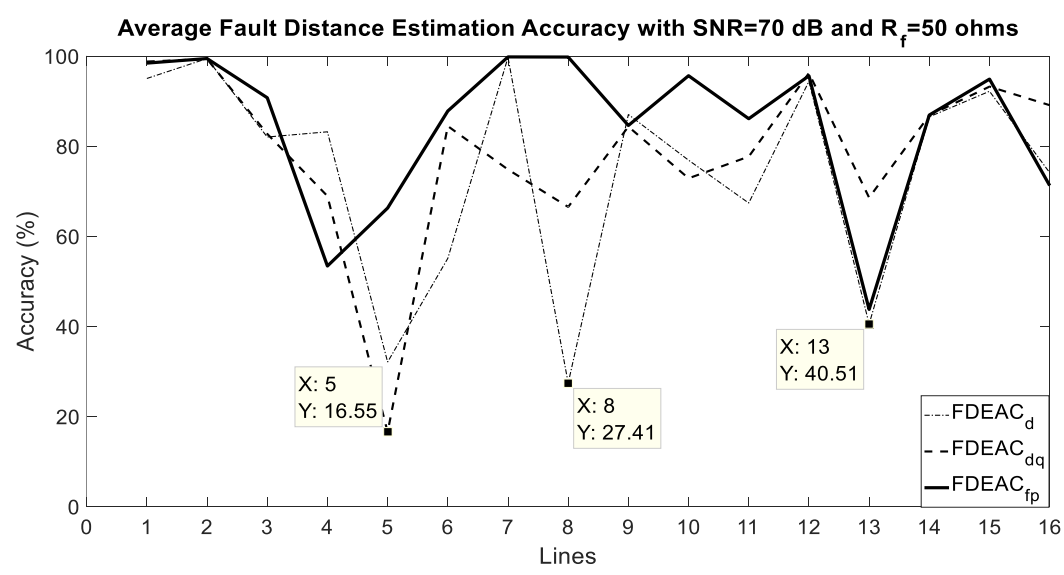

(b)

Figure 6. Average fault distance estimation accuracy for all lines with $\mathrm{SNR}=70 \mathrm{~dB}$ for (a) $R_{f}=0.001 \Omega$ and (b) $R_{f}=50 \Omega$ 
The performance of overall average estimation accuracy for all specified SNR values is illustrated in Figure 7(a) for $0.001 \mathrm{Ohm}$ minimum fault resistance and Figure 7(b) for $50 \mathrm{Ohm}$ maximum assumed fault resistance respectively. In Figure 7, The results compare the fault distance estimation accuracy all lines with the same SNR. Three lines represents the value estimated with $\mathrm{d} 0$ time delay, dq time delay and FP time delay to demonstrate the advantage of the proposed algorithm.

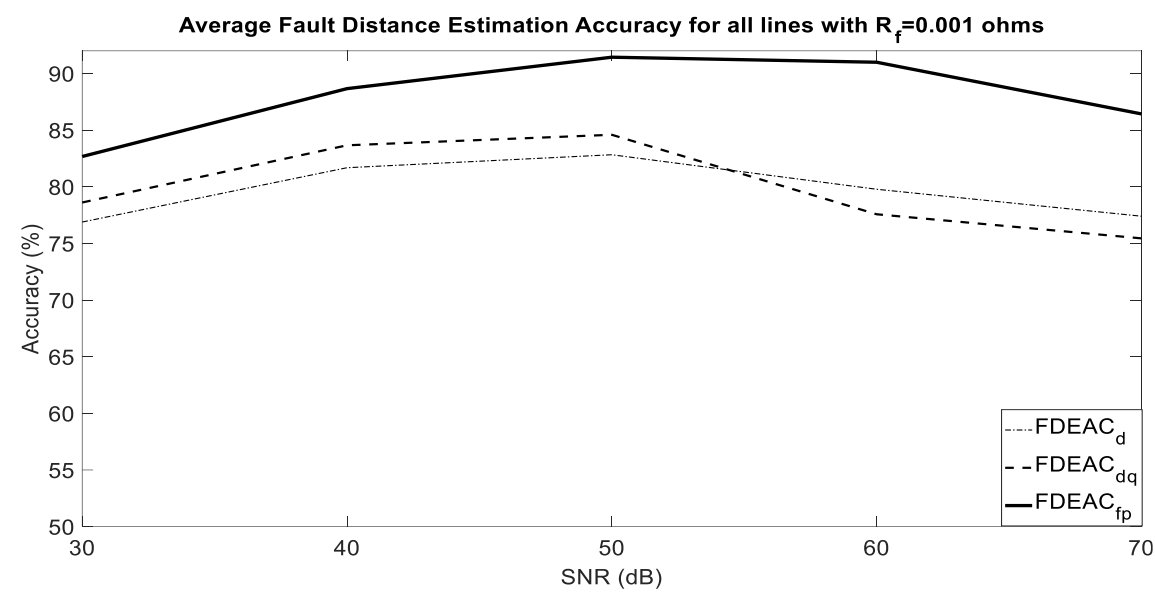

(a)

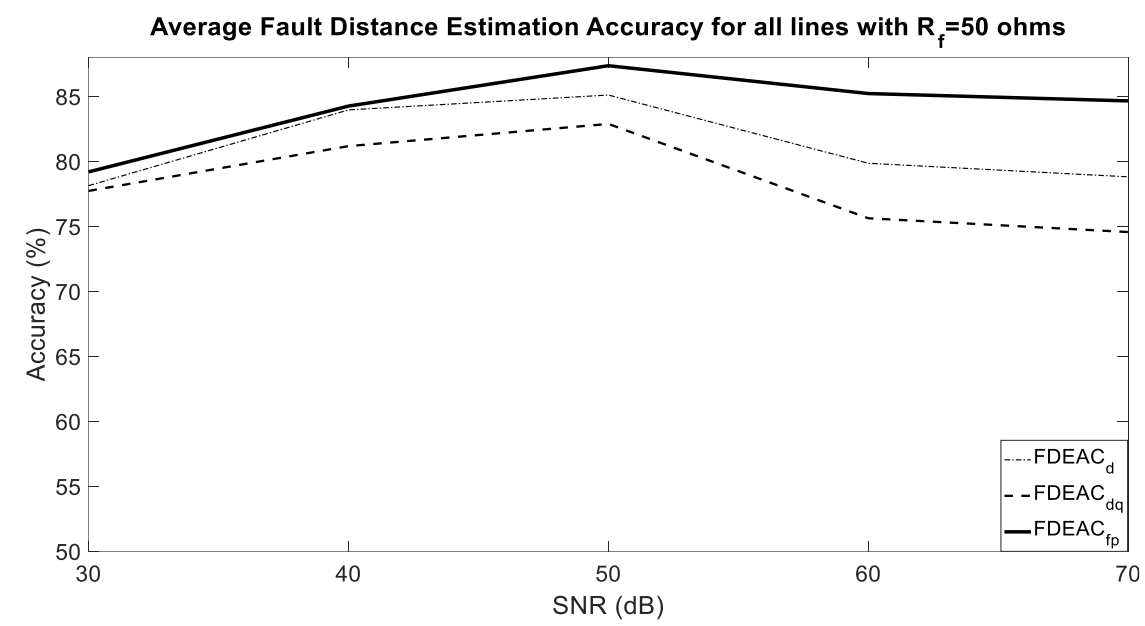

(b)

Figure 7. Overall average estimation accuracy in all lines with $\mathrm{SNR}=30$ to $70 \mathrm{~dB}$ for $(\mathrm{a}) \mathrm{R}_{\mathrm{f}}=0.001 \Omega$ and

(b) $\mathrm{R}_{\mathrm{f}}=50 \Omega$

According to the result in Figure 7, the averaged estimation accuracy of the proposed faulted phase time delay demonstrates that it gives better accuracy than conventional time delay from aerial component $\left(\Delta t_{d}\right)$ and aerial different component $\left(\Delta t_{d q}\right)$ for all the noise case. This is due to the fact that the faulted phase contains more information about the fault than the healthy line, which used in the conventional dq transformation. Hence, the proposed algorithm has demonstrated that it can accurately locate the fault in a loop transmission under the simulation even with additive noise.

\section{CONCLUSION}

This paper proposes a simple fault localization technique in loop distribution systems. The proposed technique passed through band pass filter, Karenbauer's transform, and wavelet transform criteria to estimate for fault distance by using only three phase current signals at the source bus (B1) of the test loop distribution network. The proposed algorithm uses the arriving time of faulted phase-based modal component that can give higher accuracy than that of conventional methods with the consideration of noise effects, such 
as white noise. According to detail level analysis section, using the arriving times of the component combined the three reconstructed signals using three detail level coefficients (D1, D2 and D3) can give best accuracy. The proposed algorithm shows that the faulted phase time delay() can give better accuracy than using conventional time delay() and aerial different time delay().

The algorithm performance is successfully tested with data obtained by simulations with 1650 different cases of sampled data at various situations, faulted feeders, faulted resistance, 30 to $70 \mathrm{~dB}$ SNR values and fault locations. The proposed methodology is evaluated through MATLAB/Simulation single line to ground fault in a $10 \mathrm{kV}$ grounded loop distribution system. The proposed algorithm can give fault location accuracy up to $99.7 \%$ with $30 \mathrm{~dB}$ contaminated SNR for the nearest lines from the measured terminal. Using proposed algorithm, the overall fault information accuracy can get up to $83 \%$ and overall fault distance estimation accuracy up to $93 \%$. This proposed technique can be used to implement in real data with actual fault records.

\section{ACKNOWLEDGEMENTS}

The authors would like to express her gratitude to their scholarship program, the Higher Education Research Promotion and Thailand's Education Hub for Southern Region of ASEAN Countries (TEH-AC).

\section{REFERENCES}

[1] Y. Liao, "A novel method for locating faults on distribution systems," Elect. Pow. Syst. Res., vol. 117, pp. 21-26, 2014, doi: 10.1016/j.epsr.2014.07.026.

[2] S. A. Al Kazzaz, I. Ismael, and K. K. Mohammed, "Fault detection and location of power transmission lines using intelligent distance relay," International Journal of Power Electronics and Driver System, vol. 11, no. 2, pp. 726-734, Jun. 2020, doi: 10.11591/ijpedsv11.i2pp726-734.

[3] A. C. Adewole, R. Tzoneva, and S. Behardien, "Distribution network fault section identification and fault location using wavelet entropy and neural networks," Applied Soft Computing, vol. 46, pp. 296-306, Sept. 2016, doi: 10.1016/j.asoc.2016.05.013.

[4] A. Swetapadma and A. Yadav, "A novel decision tree regression-based fault distance estimation scheme for transmission lines," IEEE Transactions on Power Delivery, vol. 32, no. 1, pp. 234-245, Feb. 2017, doi: 10.1109/TPWRD.2016.2598553.

[5] X. Deng, R. Yuan, Z. Xiao, T. Li, and K. L. L. Wang, "Fault location in loop distribution network using SVM technology," International Journal of Electrical Power \& Energy System, vol. 65, pp. 254-261, Feb. 2015, doi: 10.1016/j.ijepes.2014.10.010.

[6] F. I. Jabbar, D. Soomro, A. H. Tawafan, M. N. Abdullah, N. H. Radzi, and M. H. Baloch, "Optimization of detection of a single line to ground fault based on ABCNN algorithm," International Journal of Power Electronics and Driver System, vol. 9, no. 4, pp. 623-629, Dec. 2020, doi: 10.11591/ijai.v9.i4.pp623-629.

[7] S. S. D. Sahel and M. Boudour, "Wavelet energy moment and neural networks-based particle swarm optimisation for transmission line protection," Bulletin of Electrical Engineering and Informatics, vol. 8, no. 1, pp. 10-20, Mar. 2019, doi: 10.11591/eeiv8i1.1214.

[8] M. Daisy and R. Dashti, "Single phase fault location in electrical distribution feeder using hybrid method," Journal Energy, vol. 103, pp. 356-368, May 2016, doi: 10.1016/j.energy.2016.02.097.

[9] S. H. Mortazavi, Z. Moravej, and S. M. Shahrtash, "A searching based method for locating high impedance arcing fault in distribution networks," IEEE Transactions on Power Delivery, vol. 34, no. 2, pp. 438-447, Apr. 2019, doi: 10.1109/TPWRD.2018.2874879.

[10] M. J. S. Ramos, A. S. Bretas, D. P. Bernardon, and L. L. Pfitscher, "Distribution network HIF location: A frequency domain system model and WLS parameter estimation approach," Electrical Power Systems Research, vol. 146, pp. 170-176, May 2017, doi: 10.1016/j.epsr.2017.01.030.

[11] P. Cao, H. Shu, B. Yang, J. Dong, Y. Fang and T. Yu, "Speeded-up robust features based single-ended traveling wave fault location: a practical case study in Yunnan power grid of China," IET Generation Transmission \& Distribution, vol. 12, no. 4, pp. 886-894, Feb. 2018, doi: 10.1049/iet-gtd.2017.0735.

[12] E. E. Aker, M. L. Othman, I. Aris, N. I. Abdul Wahab, H. Hizam, and O. Emmanuel, "Transmission line fault identification and classification with integrated FACTS device using multiresolution analysis and naïve bayes classifier," International Journal of Power Electronics and Driver System, vol. 11, no. 2, pp. 907-913, Jun. 2020, doi: 10.11591/ijpedsv11.i2pp907-913.

[13] J. Ding, L. Li, Y. Zheng, C. Zhao, H. Chen, and X. Wang, "Distributed traveling-wave-based fault location without time synchronization and wave velocity error," IET Generation Transmission \& Distribution, vol. 11, no. 8, pp. 2085-2093, Jun. 2017, doi: 10.1049/iet-gtd.2016.1778.

[14] S. Shi, B. Zhu, A. Lei, and X. Dong, "Fault location for radial distribution network via topology and reclosure-generating traveling waves," IEEE Transactions on Smart Grid, vol. 10, no. 6, pp. 6404-6413, Nov. 2019, doi: 10.1109/TSG.2019.2904210.

[15] J. Sadeh, E. Bakhshizadeh, and R. Kazemzadeh, "A new fault location algorithm for radial distribution systems using modal analysis," International Journal of Power Electronics \& Driver System, vol. 45, no. 1, pp. 271-278, Feb. 2013, doi: 10.1016/j.ijepes.2012.08.053.

[16] R. Liang, F. Wang, G. Fu, X. Xue, and R. Zhou, "A general fault location method in complex power grid based on wide-area traveling wave data acquisition," International Journal of Power Electronics \& Driver System, vol. 83, pp. 213-218, Dec. 2016, doi: 10.1016/j.ijepes.2016.04.021.

[17] E. Khoudry, A. Belfqih, J. Boukherouaa, and F. Elmariami, "Traveling wave-based fault location for power transmission lines using morphological filters and clarke modal components International Journal of Electrical and Computer Engineering., vol. 10, no. 2, pp. 1122-1134, Apr. 2020, doi: 10.11591/ijecev10i2.pp1122-1134.

[18] B. K. Chaitanya and A. Yadva, "Decision tree aided traveling wave-based fault section identification and location scheme for multi-terminal transmission lines," Journal Measurement, vol. 135, pp. 312-322, Mar. 2019, doi: 10.1016/j.measurement.2018.11.069. 
[19] L. Ye, D. You, X. Yin, K. Wang, and J. Wu, “An improved fault-location method for distribution system using wavelets and support vector regression," International Journal of Power Electronics and Energy System, vol. 55, pp. 467-472, Feb. 2014, doi: 10.1016/j.ijepes.2013.09.027.

[20] Q. Jia, X. Dong, and S. Mirsaeidi, "A traveling-wave-based line protection strategy against single-line-to-ground faults in active distribution networks," International Journal of Power Electronics and Energy System, vol. 107, pp. 403-411, May 2019, doi: 10.1016/j.ijepes.2018.11.032.

[21] L. Rui, F. Guoqing, Z. Xueyuan, and X. Xue, "Fault location based on single terminal traveling wave analysis in radial distribution network," International Journal of Power Electronics and Energy System, vol. 66, pp. 160-165, Mar. 2015, doi: 10.1016/j.ijepes.2014.10.026.

[22] L. Rui, P. Nan, Y. Zhi, and F. Zare, "A novel single-phase-to-earth fault location method for distribution network based on zerosequence components distribution characteristics," International Journal of Power Electronics and Energy System, vol. 102, pp. 11-22, Nov. 2018, doi: 10.1016/j.ijepes.2018.04.015.

[23] L Rui, L. Chenglei, P. Nan, C. Menghan, and W. Fei. "Fault location for power grid based on transient traveling wave data fusion via asynchronous voltage measurements," International Journal of Power Electronics and Energy System, vol. 93, pp. 426-439, Dec. 2017, doi: 10.1016/j.ijepes.2017.06.002.

[24] C. Zhou, Q. Shu, and X. Han, "A single phase earth fault location scheme for distribution feeder on the basis of the difference of zero mode traveling waves," International Journal of Power Electronics and Energy System, pp. 1-9, Dec. 2016, doi: 10.1002/etep.2298.

[25] S. Myint and W. Wichakool, "A traveling wave-based fault section and fault distance estimation algorithm for grounded distribution systems," in 2019 IEEE PES GTD Grand International Conference and Exposition. Asia, Bangkok, Thailand, pp. 472-477, Mar. 2019, doi: 10.1109/GTDAsia.2019.8715933.

[26] S. Myint and W. Wichakool, "Fault type identification method based on wavelet detail coefficients of modal current components," in 2018 IEEE $5^{\text {th }}$ International Conference on Smart Instrumentation Measurement and Application, Songkhla, Thailand, pp. 1-6, Nov. 2018, doi: 10.1109/ICSIMA.2018.8688796.

\section{BIOGRAPHIES OF AUTHORS}

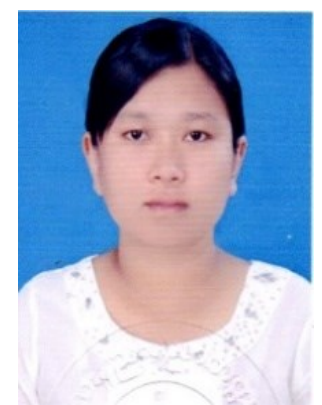

Shwe Myint (iD If SC P received the B.E and M.E degrees in Electrical Engineering from Mandalay Technological University (MTU), Mandalay, Myanmar in 2006 and 2014 respectively. She is currently pursuing the Ph.D. degree program in Electrical Engineering Department at Prince of Songkla University, Hatyai Campus, Songkhla, Thailand. Her current research interests include power system analysis, renewable energy, signal processing and machine learning. She can be contacted at email: 5910130043@psu.ac.th.

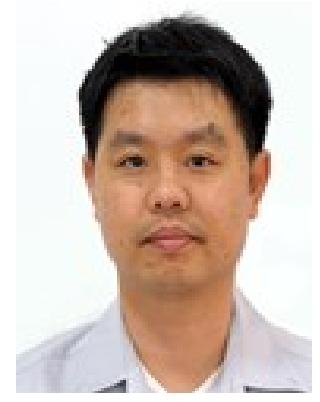

Warit Wichakool (iD) 8d SC P received the Ph.D. degree in Electrical Engineering and Computer Science from the Massachusetts Institute of Technology, Cambridge, MA, USA, in 2011. He is currently working at Electrical Engineering Department at Prince of Songkla University, Hatyai Campus, Songkhla, Thailand. His current research interests include power system analysis, power converter design, power electronics circuits and applications and renewable energy. He can be contacted at email: warit.wi@psu.ac.th. 